\title{
ANOVA parameters influence in LCF experimental data and simulation results
}

\author{
C. Delprete, R. Sesana ${ }^{\mathrm{a}}$, A. Vercelli \\ Dipartimento di Meccanica, Politecnico di Torino, Corso Duca degli Abruzzi 24, 10129 Torino Italy
}

\section{Abstract}

The virtual design of components undergoing thermo mechanical fatigue (TMF) and plastic strains is usually run in many phases. The numerical finite element method gives a useful instrument which becomes increasingly effective as the geometrical and numerical modelling gets more accurate. The constitutive model definition plays an important role in the effectiveness of the numerical simulation $[1,2]$ as, for example, shown in Figure 1. In this picture it is shown how a good cyclic plasticity constitutive model can simulate a cyclic load experiment.

The component life estimation is the subsequent phase and it needs complex damage and life estimation models [3-5] which take into account of several parameters and phenomena contributing to damage and life duration. The calibration of these constitutive and damage models requires an accurate testing activity.

In the present paper the main topic of the research activity is to investigate whether the parameters, which result to be influent in the experimental activity, influence the numerical simulations, thus defining the effectiveness of the models in taking into account of all the phenomena actually influencing the life of the component.

To obtain this aim a procedure to tune the parameters needed to estimate the life of mechanical components undergoing TMF and plastic strains is presented for commercial steel. This procedure aims to be easy and to allow calibrating both material constitutive model (for the numerical structural simulation) and the damage and life model (for life assessment).

The procedure has been applied to specimens. The experimental activity has been developed on three sets of tests run at several temperatures: static tests, high cycle fatigue (HCF) tests, low cycle fatigue (LCF) tests.

The numerical structural FEM simulations have been run on a commercial non linear solver, ABAQUS®6.8. The simulations replied the experimental tests.

The stress, strain, thermal results from the thermo structural FEM simulations have been used to assess specimens life by means of several damage models implemented in two dedicated softwares. One of them is commercial life estimation software and the second one has been developed by the present research group.

The life estimations results obtained from the two softwares and from the damage models have been compared with experimental duration in different test conditions. A statistical analysis of life estimation results allowed evaluating the effectiveness of the procedure. 

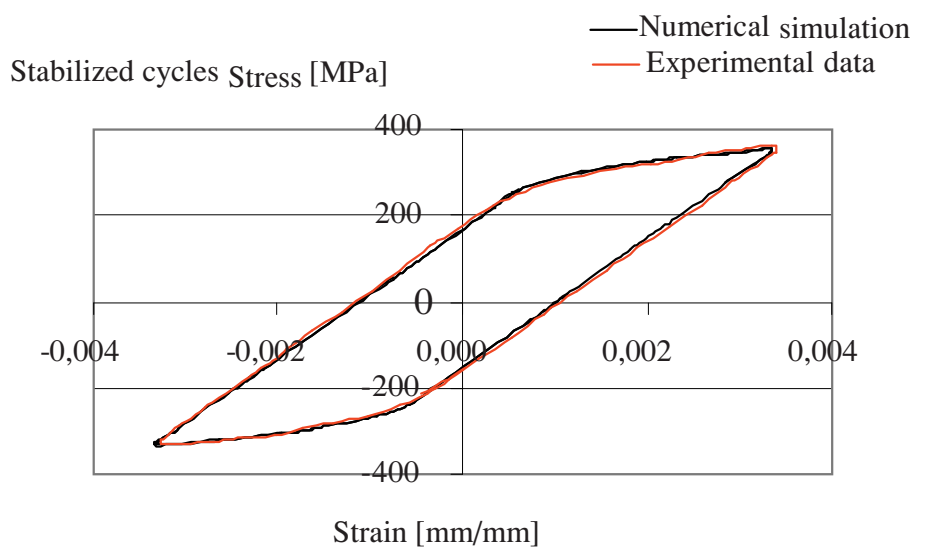

Fig. 1. symmetrical displacement cyclic test: comparison of experimental and simulated data results.

\section{References}

1. H. Hubel, Nuclear Engineering and Design, 162, 55-65 (1996)

2. B.K. Chun, J.T. Jinn, J.K. Lee, Int. J. of Plasticity, 18 (5-6), 571-595 (2002)

3. Z. Mroz, A. Seweryn, ASME Mechanics Conference, Evanston, Illinois, USA, (1997)

4. G.Z. Voyiadjis, J.W. Woody Ju, J.L. Chaboche, Damage Mechanics in Engineering Materials, Elsevier, Amsterdam, NL, (1998)

5. J. Lemaitre, J.L. Chaboche, Mechanics of Solid Materials, Cambridge University Press, New York, USA, (2000) 